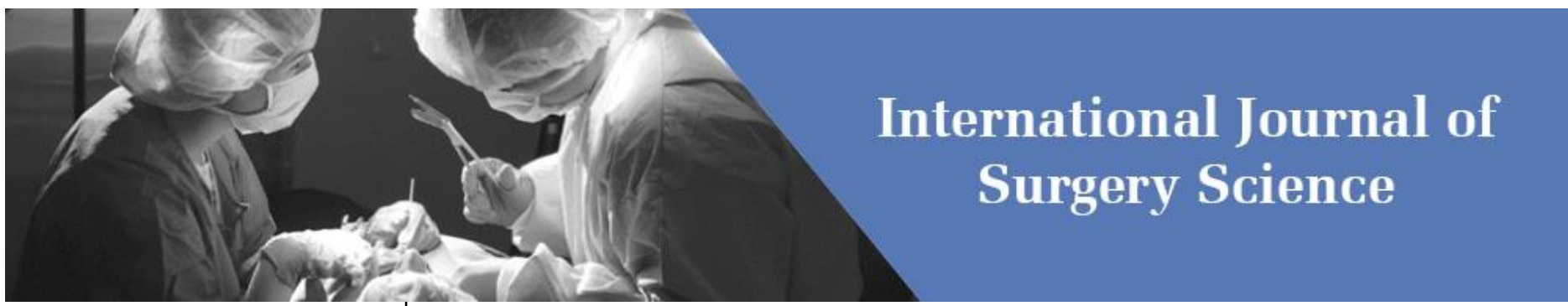

E-ISSN: 2616-3470

P-ISSN: 2616-3462

(C) Surgery Science

www.surgeryscience.com

2020; 4(1): 257-260

Received: 04-11-2019

Accepted: 08-12-2019

Dr. Deepak Naik

Assistant Professor, Department of

General Surgery, Jss Medical

College, Jssaher, Mysuru,

Karnataka, India

Dr. Lavanya Raghupathi

Junior Resident, Department of

General Surgery, Jss Medical

College, Jssaher, Mysuru,

Karnataka, India

Dr. Ashwini Kumar Choudhary Junior Resident, Department of General Surgery, Jss Medical College, Jssaher, Mysuru,

Karnataka, India
Corresponding Author: Dr. Lavanya Raghupathi Junior Resident, Department of General Surgery, Jss Medical College, Jssaher, Mysuru, Karnataka, India

\section{Role of neutrophil lymphocyte ratio in predicting lymphovascular invasion in carcinoma breast}

\author{
Dr. Deepak Naik, Dr. Lavanya Raghupathi and Dr. Ashwini Kumar \\ Choudhary
}

DOI: https://doi.org/10.33545/surgery.2020.v4.i1e.343

\section{Abstract}

Introduction: Inflammation is a protective response which involves immune cells, blood vessels, and molecular mediators. Neutrophils and lymphocytes help in healing and regeneration of damaged tissues and also fight infections. Neutrophil to lymphocyte ratio (NLR) is used as a pointer or marker for inflammation both clinical and subclinical. The presence of lymphovascular invasion (LVI) is understood as or points to increased risk of axillary lymph node and distant metastases.

Aims and Objectives: To compare neutrophil to lymphocyte ratio [NLR] with lymphovascular invasion in carcinoma breast.

Methodology: A descriptive study was conducted at JSS Hospital Mysuru over a period of two years comprising of 100 patients diagnosed as carcinoma breast and results were drawn. All demographic data was tabulated and measured as proportion, mean, standard deviation and the inferential statistics were obtained using Chi-square and Cramer's V tests.

Results: $65 \%$ of carcinoma breast cases in our study had lymphovascular invasion. The percentage of lymphovascular invasion increased with increase in NLR progressively with 22.2 percent in category A to 91.8 percent in category $\mathrm{D}$.

Conclusion: Increase in neutrophil to lymphocyte ratio is associated with increased incidence of lymphovascular invasion in carcinoma breast.

Keywords: Neutrophils, Lymphocytes, Neutrophil Lymphocyte ratio (NLR) Lymphovascular invasion (LVI), Carcinoma breast

\section{Introduction}

Inflammation is derived from Latin word "inflammation" which is a part of the complex biological response of cells and tissues of body to advert stimuli, such as microbes, pathogens, foreign cells, or irritants, ${ }^{[1]}$ and is a protective response which involves immune cells, blood vessels, and molecular mediators. The cells of the immune system are of several types as lymphocytes (T-cells, B-cells and NK cells), neutrophils, and monocytes/macrophages. These are all types of WBC's.

Neutrophils are a type of white blood cell that helps in healing and regeneration of damaged tissues and also fight infections. Blood levels of neutrophils increase naturally in response to infections, injuries, and other types of stress.

Lymphocytes are also one of the subtypes of a white blood cell in our immune system. Lymphocytes comprises of $\mathrm{T}$ cells, Natural killer cells and B cells (for humoral, antibodydriven adaptive immunity) which are mostly present in lymph, hence the name "lymphocyte".

Neutrophil to lymphocyte ratio (NLR) is used as a pointer or marker for inflammation both clinical and subclinical. It is calculated by dividing the number of neutrophils by number of lymphocytes, usually from peripheral blood sample but sometimes also from cells that infiltrate tissue, such as tumor ${ }^{[2]}$.

Lymphovascular invasion (LVI) is described as the presence of tumor cells within a definite endothelial-lined space (lymphatics or blood vessels) in the breast surrounding the actual invasive lump. The presence of this is understood as or points to increased risk of axillary lymph node and distant metastases ${ }^{[3]}$.

It is well known that systemic inflammatory factors play a significant role in progression of cancer $^{[4,5]}$. The micro-environment of the tumour regulated by these cells is clearly related to 
Cancer progression. Systemic inflammatory factors are reflected by changes of peripheral leukocyte, lymphocytes, neutrophils, monocytes and platelets and can reflect the tumor micro environment indirectly in cancer patients.

Breast cancer is a heterogeneous complex of diseases that has different subtypes which have different treatment responses and outcomes clinically.

Tumor characteristics and host response, including the inflammatory response, both contribute to the clinical outcomes of cancer patients ${ }^{[6]}$. Histopathological analysis of tumors has shown tumor infiltration by inflammatory and lymphocytic cells.

\section{Objectives \\ Primary Objective}

To compare neutrophil to lymphocyte [NLR] with lymphovascular invasion in carcinoma breast.

\section{Methodology}

a. Study Design: Descriptive Study

b. Study place: JSS Hospital Department of Surgery

c. Study Duration: January 2017 to December 2019

d. Sample size: Sample Size is considered as 100

e. Sampling technique and study population: Convenient sample. All consecutive cases coming to surgical department.

\section{f. Inclusion criteria}

All proven cases of Carcinoma Breast who have under gone modified radical mastectomy.

\section{g. Exclusion criteria}

1. Benign breast disease

2. Patients with bleeding diathesis

3. Patients unwilling for modified radical mastectomy

4. Patients not willing to be part of study.

Study Assessments of end points - To assess lymphovascular invasion in carcinoma breast and compare the same with NLR.

\section{Statistical methods applied \\ Data Analysis}

All demographic data is summarized as proportion, mean, median, standard deviation and tabulated. All the measurements are done using SPSS version 21 and EPI-Info version 7

\section{Descriptive statistics}

The Descriptive procedure displays univariate summary statistics for several variables in a single table and calculates standardized values (z scores). Variables can be ordered by the size of their means (in ascending or descending order), alphabetically, or by the order in which the researcher specifies.

Descriptive statistics included

Mean,

Median,

Standard deviation,

Frequency and

Percent

\section{Inferential statistics \\ Chi-Square Test}

The Chi-Square Test procedure tabulates a variable into categories and computes a chi-square statistic. This goodness-offit test compares the observed and expected frequencies in each category to test either that all categories contain the same proportion of values or that each category contains a userspecified proportion of values.

\section{Crosstabs (Cramer's V)}

The Crosstabs procedure forms two-way and multiway tables and provides a variety of tests and measures of association for two-way tables. The structure of the table and whether categories are ordered determine what test or measure to use. Cramer's V test was employed in the present study.

\section{Results}

Neutrophil lymphocyte ratio [NLR] was obtained for all carcinoma breast cases and a median of 2.03 was taken for all values, the lowest value being 1.27 and maximum being 3.34 . Based on this various categories of NLR were formed in this study-

1. Category A having NLR of less than equal to 1.5

2. Category B having NLR of 1.51-2.0

3. Category $C$ having NLR of 2.01-2.5

4. Category D having NLR of more than 2.5

Platelet count was also taken into account in the study and a median value of 2.52 was obtained and accordingly various categories of platelets were formed

1. Category X- 1 to 2 lakhs/cumm

2. Category Y-2 to 2.52 lakhs/cumm

3. Category Z- more than 2.52 lakhs /cumm

Table 1: Platelets VS NLR

\begin{tabular}{|c|c|c|c|c|c|c|c|}
\hline & \multicolumn{4}{|c|}{ NLR } & \multirow{2}{*}{ Total } \\
\hline & & & $\mathbf{A}$ & B & C & D & \\
\hline \multirow{6}{*}{ platelets } & \multirow{2}{*}{$\mathrm{X}$} & Count & 1 & $\mathbf{0}$ & 1 & 2 & 4 \\
\hline & & $\%$ within NLR & $3.7 \%$ & $0.0 \%$ & $6.7 \%$ & $4.1 \%$ & $4.0 \%$ \\
\hline & \multirow[b]{2}{*}{$\mathrm{Y}$} & Count & 8 & 3 & 3 & 7 & 21 \\
\hline & & $\%$ within NLR & $29.6 \%$ & $33.3 \%$ & $20.0 \%$ & $14.3 \%$ & $21.0 \%$ \\
\hline & \multirow{2}{*}{$\mathrm{Z}$} & Count & 18 & 6 & 11 & 40 & 75 \\
\hline & & $\%$ within NLR & $66.7 \%$ & $66.7 \%$ & $73.3 \%$ & $81.6 \%$ & $75.0 \%$ \\
\hline \multirow{2}{*}{\multicolumn{2}{|c|}{ Horat }} & Count & 27 & 9 & 15 & 49 & 100 \\
\hline & & $\%$ within NLR & $100.0 \%$ & $100.0 \%$ & $100.0 \%$ & $100.0 \%$ & $100.0 \%$ \\
\hline
\end{tabular}

In our study it was observed that a lower NLR eg in Category A -66.7 percent cases had value of platelets more than 2.5 lakhs/c $\mathrm{mm}$, in category $\mathrm{B}$ this was again 66.7 percent, in category $\mathrm{C}$ it increased to 73.3 percent whereas in category $\mathrm{D}$ this further increased to 81.6 percent.

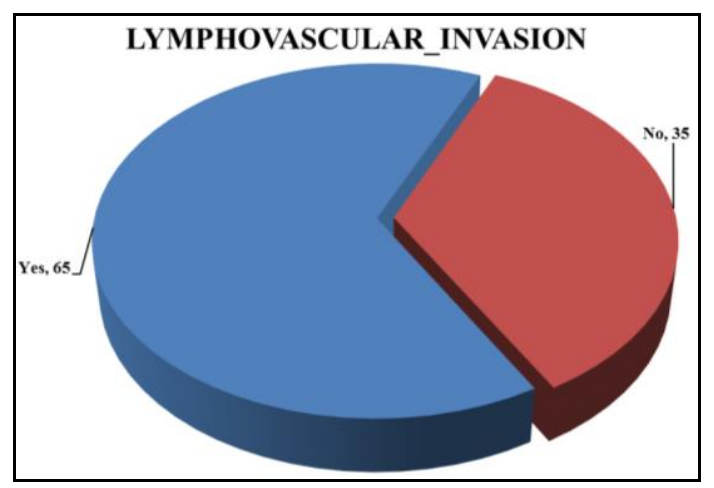

Pie Chart 1: Lymphovascular invasion

Out of a total 100 cases of carcinoma breast lymphovascular invasion was seen in 65 case while in 35 cases there was no lymphovascular invasion 
Table 2: Lymphovascular invasion VS NLR

\begin{tabular}{|c|c|c|c|c|c|c|c|}
\hline & \multicolumn{4}{|c|}{ NLR } & \multirow{2}{*}{ Total } \\
\hline & & & $\mathbf{A}$ & B & $\mathbf{C}$ & D & \\
\hline \multirow{4}{*}{ Lymphovascular invasion } & \multirow{2}{*}{ Yes } & Count & 6 & 3 & 11 & 45 & 65 \\
\hline & & $\%$ within NLR & $22.2 \%$ & $33.3 \%$ & $73.3 \%$ & $91.8 \%$ & $65.0 \%$ \\
\hline & \multirow{2}{*}{ No } & Count & 21 & 6 & 4 & 4 & 35 \\
\hline & & $\%$ within NLR & $77.8 \%$ & $66.7 \%$ & $26.7 \%$ & $8.2 \%$ & $35.0 \%$ \\
\hline \multirow{2}{*}{\multicolumn{2}{|c|}{ Total }} & Count & 27 & 9 & 15 & 49 & 100 \\
\hline & & $\%$ within NLR & $100.0 \%$ & $100.0 \%$ & $100.0 \%$ & $100.0 \%$ & $100.0 \%$ \\
\hline
\end{tabular}

In category A i.e. NLR less than or equal to 1.5 Lymphovascular invasion was present in $22.2 \%$ cases whereas in $77.8 \%$ cases there was no lymphovascular invasion

In category B $33.3 \%$ cases had lymphovascular invasion while in $66.7 \%$ cases there was no lymphovascular invasion

In category $\mathrm{C} 73.7 \%$ cases had lymphovascular invasion while 26.7 percent cases of carcinoma breast had no lymphovascular invasion.

In category D $91.8 \%$ cases had lymphovascular invasion whereas in $8.2 \%$ cases there was no lymphovascular invasion.

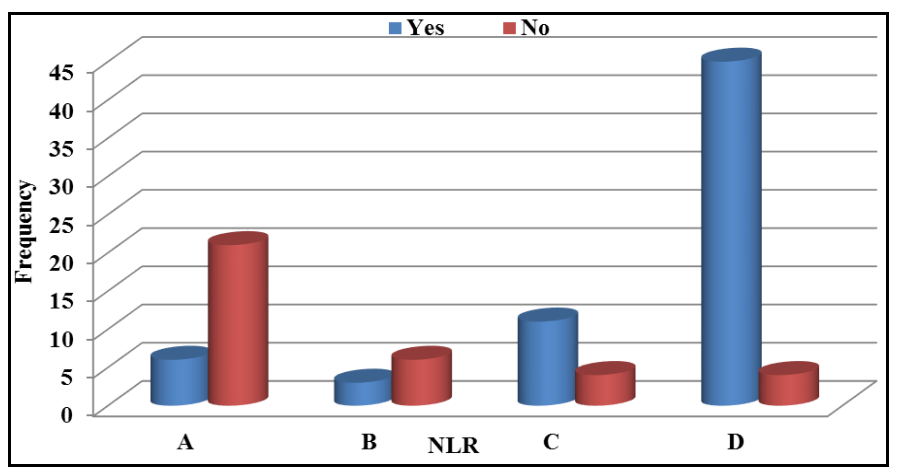

Fig 1: Bar diagram - NLR VS Lymphovascular invasion

\section{Discussion}

The NLR, obtained by the number of neutrophils and lymphocytes, is frequently used as a tool in evaluating systemic inflammation. Many studies have corroborated that NLR is related with the prognosis in breast, colorectal, gastric, pancreas, hepatocellular, and renal cancers which is evident by the fact that neutrophils promote tumor cell proliferation, angiogenesis, and distant metastasis. Lymphocytes also play a central role in the immune response to various tumor cells. This leads one to predict that NLR is associated with prognosis ${ }^{[7,8,9]}$.

Platelets are essential components of hemostasis and also play a pivotal role in the tumor microenvironment. Platelet count has been reported to have predictive value in different cancer types.

Increased risk of thrombosis as well as increased platelet activation has been observed particularly in women with breast cancer, but until recently, these clinical observations have been considered a paraneoplastic phenomenon ${ }^{[10,11,12,13,14]}$.

Thrombocytosis can be an early indicator of cancer as a generalized entity which is evident by the fact that in women with ovarian adenocarcinoma prevalence of thrombocytosis (average platelet count $=558 \times 106 / 1$ ) at the time of diagnosis is more. More importantly, paraneoplastic thrombocytosis in these patients is found to be associated with an advanced stage and shortened survival ${ }^{[15]}$.

However according to some studies, preoperative thrombocytosis is not a prognostic indicator of survival in colorectal cancer ${ }^{[16]}$.

The literature on thrombocytosis and breast cancer is limited and has high controversies. In breast cancer particularly high interleukin-6 (IL-6) levels and platelet counts have been reported, in patients with metastatic breast cancer as compared to those with localized disease ${ }^{[17]}$.

"A recent publication has shed some light to the pathogenesis of thrombocytosis in cancer ${ }^{[8]}$ and confirmed previous reports on the role of cytokines and in particular of IL-6." [18]

According to another study "Thrombocytosis (more than $400 \times$ $109 / \mathrm{L}$ ) was evaluated as a prognostic factor in an extensive series of 1513 patients with localized colorectal cancer that had undergone surgery" [19] which signified its importance in predicting the prognosis.

In our study 100 cases of carcinoma breast were included in the study and modified radical mastectomy is done for all cases as per standard guidelines. After the surgery all the specimens were subjected to histopathology and presence of lymphovacular invasion was noted for all cases which was compared to various categories of NLR formed considering 2.03 as median value.

65 percent of cases had lymphovascular invasion, the results were tabulated and compared. Lymphovascular invasion progressively increased from 22.2 percent in category A to 33.3 percent in category B to 73.7 percent in category $\mathrm{C}$ to 91.8 percent in category $\mathrm{D}$. A value of more than the median value i.e. NLR more than 2.03 was found to be significantly associated with lymphovascular invasion with a $\mathrm{p}$ value of 0.03 .

Also it was noted in the study that increase in number of platelets is associated with a corresponding increase in NLR, thereby making thrombocytosis a significant marker of lymphovascular invasion.

\section{Conclusion}

- Increase in platelets is observed in with an increase in NLR.

- Thrombocytosis is a marker of lymphovascular invasion.

- Increase in NLR signifies lymphovascular invasion and thus an advance stage of carcinoma breast.

\section{References}

1. Ferrero-Miliani L, Nielsen OH, Andersen PS, Girardin SE. Chronic inflammation: importance of NOD2 and NALP3 in interleukin-1beta generation. Clin Exp Immunol. 2007; 147(2):227-235.

2. Wang J, Jia $\mathrm{Y}$, Wang $\mathrm{N}$ et al. The clinical significance of tumor-infiltrating neutrophils and neutrophil-to-CD8+ lymphocyte ratio in patients with resectable esophageal squamous cell carcinoma. J Transl Med. 2014; 12:7. Published 2014 Jan 7. doi:10.1186/1479-5876-12-7

3. Ejlertsen B, Jensen MB, Rank F et al. Population-based study of peritumoral lympho vascular invasion and outcome among patients with operable breast cancer. J Nat 1 Cancer Inst. 2009; 101:729-35.

4. Coussens LM, Werb ZJN. Inflammation and cancer. 2002; 420(6917):860.

5. Sandler RS, Halabi S, Baron JA, Budinger S, Paskett E, Keresztes $\mathrm{R}$ et al. A randomized trial of aspirin to prevent colorectal adenomas in patients with previous colorectal 
cancer. 2003; 348(10):883-90.

6. Yersal O, Barutca S. Biological subtypes of breast cancer: Prognostic and therapeutic implications. World journal of clinical oncology. 2014; 5(3):412.

7. Gomez D, Farid S, Malik HZ, Young AL, Toogood GJ, Lodge JP et al. Preoperative neutrophil-to-lymphocyte ratio as a prognostic predictor after curative resection for hepatocellular carcinoma. World J Surg. 2008; 32(8):175762.

8. Ohno Y, Nakashima J, Ohori M, Gondo T, Hatano T, Tachibana M. Followup of neutrophil-to-lymphocyte ratio and recurrence of clear cell renal cell carcinoma. J Urol. 2012; 187(2):411-7.

9. Koh CH, Bhoo-Pathy N, Ng KL, Jabir RS, Tan GH, See $\mathrm{MH}$ et al. Utility of pre-treatment neutrophil lymphocyte ratio and platelet-lymphocyte ratio as prognostic factors in breast cancer. Br J Cancer. 2015; 113(1):150-8.

10. Chew HK, Wun T, Harvey DJ, Zhou H, White RH: Incidence of venous thromboembolism and the impact on survival in breast cancer patients. J Clin Oncol. 2007; 25:70-76.

11. Caine GJ, Stonelake PS, Rea D, Lip GY: Coagulopathic complications in breast cancer. Cancer. 2003; 98:15781586.

12. Caine GJ, Lip GY, Stonelake PS, Ryan P, Blann AD: Platelet activation, coagulation and angiogenesis in breast and prostate carcinoma. Thromb Haemost.2004, 92:185-190.

13. Ferriere JP, Bernard D, Legros M, Chassagne J, Chollet P, Gaillard $\mathrm{G}$ et al: beta-Thromboglobulin in patients with breast cancer. Am J Hematol. 1985; 19:47-53.

14. Alonso-Escolano D, Stron gin AY, Chung AW, Deryugina EI, Radomski MW: Membrane type-1 matrix metalloproteinase stimulates tumour cell induced platelet aggregation: role of receptor glycoproteins. Br J Pharmacol. 2004; 141:241-252.

15. Stone RL, Nick AM, McNeish IA, Balkwill F, Han HD, Bottsford-Miller J et al: Paraneoplastic thrombocytosis in ovarian cancer. New Engl. J Med. 2012; 366:610-618,

16. Nyasavajjala SM, Runau F, Datta S, Annette H, Shaw AG Lund JN: Is there a role for pre-operative thrombocytosis in the management of colorectal cancer? Int. J Surg. 2010; 8:36-438.

17. Benoy I, Salgado R, Colpaert C, Weytjens R, Vermeulen PB, Dirix LY: Serum interleukin 6, plasma VEGF, serum VEGF, and platelet load in breast cancer patients. Clin Breast Cancer. 2002; 2:311-315.

18. Alexandrakis MG, Passam FH, Moschandrea IA, Christophoridou AV, Pappa CA et al. Levels of serum cytokines and acute phase proteins in patients with essential and cancer-related thrombocytosis. Am J clinoncol. 2003; 26:135-140.

19. Wan S, Lai Y, Myers RE, Li B, Hyslop T, London J et al. Preoperative platelet count associates with survival and distant metastasis in surgically resected colorectal cancer patients. JGastrointest Cancer. 2013; 44:293-304. 